\title{
Investigación médica en Latinoamérica
}

\section{Medical research in Latin America}

\author{
Catalina Quilindo ${ }^{1}$
}

Recientemente se han publicado artículos originales sobre la producción y visibilidad científico-académica de la Facultad de Ciencias de la Salud de la Universidad del Cauca durante los ańos 2010-2016 $(1,2)$. En su conclusión se afirma que los productos resultantes de proyectos de investigación y su consecuente publicación son escasos. Entre los principales problemas que se encuentran están: el tipo de idioma de publicación, escaso apoyo financiero, falta de tiempo, desconocimiento en la metodología de la investigación, y principalmente la carencia de una cultura institucional que tenga la investigación como una tarea incorporada en su desempeńo habitual (2).

Históricamente los médicos han comenzado sus inicios en la investigación con la finalidad de resolver los problemas que enfrentan en su práctica clínica cotidiana. Sin embargo, es necesario que el profesional cuente con bases metodológicas suficientes para desarrollar este proceso. Por tanto, es importante estimular la formación investigativa con el método científico riguroso desde los inicios de la formación académica $(3,4)$.

Al analizar el incremento exponencial de publicaciones existentes provenientes de la ciencia internacional, es inevitable que la mayoría sean escritas en el primer mundo (5), donde seguramente ya han superado muchas de las barreras nombradas previamente.

En Latinoamérica, durante el periodo 1996-2010 se produjeron 147.710 documentos totales en investigación médica de los cuales 81.230 documentos fueron publicados en Brasil ubicándose como líder en Latinoamérica en cuanto a producción científica, seguido de países como México, Argentina y Chile. Del mismo modo, Brasil lidera la región en el número de documentos citables producidos, citas recibidas y contribución porcentual a la producción mundial de documentos (6).

1 Universidad Federal de Minas Gerais, Maestranda en Investigación Clínica. Belo Horizonte, Brasil. 
Este gigantesco país sudamericano cuenta con una Fundación de Amparo a la Investigación (FAPESP) (Fundaçăo de Amparo ŕ Pesquisa do Estado de Săo Paulo) el cual está vinculado a la Secretaria de desarrollo económico de su estado, y es el principal órgano de formación científica y metodológica del país. Posee además auxilios financieros destinados a los estudiantes de pregrado y posgrado no solo en medicina si no en todas las áreas del conocimiento con el objetivo de impulsar el desarrollo en investigación.

La predominancia de producción científica brasilera es resultado de su gran población (208 millones de habitantes) y su poderío económico (PBI de 3.216 miles de millones de USD para el ańo 2017), lo cual es un factor determinante en su posición de líder en América del Sur y Latinoamérica (6).

Sin embargo hay acciones como los semilleros, grupos de investigación y las redes de colaboración internacional que son impotantes para sobrellevar las barreras descritas en el artículo mencionado. Una de las últimas, es la Colaboración Cochrane y la Facultad Ciencias de la Salud de la Universidad del Cauca se encuentra oficialmente registrada como Centro afiliado en la Colombia. Estos centros y las acciones de estudiantes y profesores, apoyadas por las instituciones, proveen la oportunidad de impulsar la labor investigativa a nivel local, permitir y fortalecer las capacidades y actitudes en los futuros investigadores, lo cual optimizará la calidad y la proyección académica institucional.

\section{REFERENCIAS}

1. Arroyo AE, Quilindo C, Diago JL, Vera-Montoya M, Delgado-Noguera M, Calvache JA. Producción académica por departamentos de la Facultad Ciencias de la Salud, Universidad del Cauca, 2010-2016. Revista de la Facultad de Ciencias de la Salud de la Universidad del Cauca. 2016;18(1):10-7.

2. Quilindo C, Calvache JA, Delgado-Noguera M. Producción y visibilidad científicoacadémica de la Facultad de Ciencias de la Salud de la Universidad del Cauca. Revista de la Facultad de Medicina. 2018;66(4),557-563.

3. Delgado-Noguera M. "Pregunta estructurada y búsqueda de la literatura: el primer paso en la práctica de la Medicina Basada en la Evidencia”. Revista de la Facultad de Ciencias de la Salud de la Universidad del Cauca. 2010;12(4):45-49.

4. Delgado-Noguera M. "David Sackett y la Medicina Basada en la Evidencia". Revista de la Facultad de Ciencias de la Salud de la Universidad del Cauca. 2015;17(3):8-9.

5. Lopez AF, Sanz-Valero J, and Culebras-FernándezJM. "Publicar en castellano, o en cualquier otro idioma que no sea inglés, negativo para el factor de impacto y citaciones." Journal of Negative and No Positive Results: JONNPR. 2016;1.2:65-70.

6. Mc Loughlin, Santiago; Rodriguez Granillo, Gaston Alfredo; Análisis de la producción científica latinoamericana en medicina; Colegio Argentino de Cardioangiólogos Intervencionistas; Revista Argentina de Cardioangiología Intervencionista.2013;3(3):164-169 